\title{
Association between sleep, eating behaviours, cardiovascular risk factors and emotional states in an overweight sample
}

\author{
S. M. Watson ${ }^{1}$, J. V. Woodside ${ }^{1}$, S. J. Hunter ${ }^{3}$, K. M. Appleton ${ }^{2}$, Young I. S. ${ }^{1}$ and M. C. McKinley ${ }^{1}$ \\ ${ }^{1}$ Centre for Public Health, Queen's University Belfast, Belfast BT12 6BJ, ${ }^{2}$ School of Psychology, \\ Queen's University Belfast, Belfast BT9 5BP and ${ }^{3}$ Regional Centre for Endocrinology and Diabetes, \\ Royal Victoria Hospital, Belfast BT12 6BA, UK
}

A relationship between obesity and poor sleep quality has been well documented in the literature ${ }^{(1)}$. Both conditions can, independently, have detrimental effects on physical and mental well being ${ }^{(2-3)}$. Few studies have examined both conditions simultaneously in relation to other parameters of health and behaviour. The objective of this study was to examine the association between sleep quality, eating behaviours, cardiovascular disease (CVD) risk factors and emotional states in an overweight/obese sample of adults (median age 52.0 years).

The analyses are based on data collected from 62 (29 males and 33 females) overweight and obese participants recruited for a webbased weight loss study. Sleep quality was measured by the Pittsburgh Sleep Quality Index (PSQI); eating behaviours were measured by the Dutch Eating Behaviour Questionnaire (DEBQ); emotional state was measured by the Depression, Stress and Anxiety scale (DASS-21); self esteem was measured by the Rosenberg Self Esteem scale and quality of life was assessed by the Impact of Weight on Quality of Life scale (IWQOL). Measured CVD risk factors included body mass index (BMI), waist hip ratio (WHR), body fat \%, blood pressure and pulse wave velocity (PWV).

PSQI score was significantly correlated with emotional eating $(r=0.41, P=0.001)$, QOL $(r=-0.43, P=0.001)$, self esteem $(r=-0.56, P=<0.001)$, depression $(r=0.62, P=<0.001)$, stress $(r=0.43, P=<0.001)$ and anxiety $(r=0.36, P=0.004)$. As shown in the table below, participants with poor sleep quality, compared to those with good sleep quality, were more likely to report higher levels of stress, anxiety and depression, have a lower self esteem and QOL score and were more likely to eat in response to their emotions and external influences. There was no significant association between CVD risk factors and sleep quality, with the exception of PWV which was significantly higher in good sleepers. A trend towards increased body fat \% in poor sleepers compared to good sleepers was observed $(P=0.06)$. Chi-square tests were used to examine the occurrence of the risk factors listed below with the occurrence of poor sleep quality. Participants with poor sleep quality were significantly more likely to be classified as being depressed $\left(\chi^{2}=19.8, \mathrm{df}=1, P=<0.001\right)$, stressed $\left(\chi^{2}=7.7, \mathrm{df}=1, P=0.006\right)$ and having low self esteem $\left(\chi^{2}=5.0, \mathrm{df}=1, P=0.04\right)$.

\begin{tabular}{|c|c|c|c|c|c|c|}
\hline \multirow[b]{2}{*}{ Health Parameters } & \multicolumn{2}{|c|}{ Total $(n=62)$} & \multicolumn{2}{|c|}{ Good Sleepers (PSQI Score $\leq 5)(n=26)$} & \multicolumn{2}{|c|}{ Poor sleepers (PSQI Score >5) $(n=36)$} \\
\hline & Median & IQ range & Median & IQ range & Median & IQ range \\
\hline BMI $\left(\mathrm{kg} / \mathrm{m}^{2}\right)$ & 33 & $30.2-34.6$ & 32.8 & $30.7-42.9$ & 33 & $30.7-34.7$ \\
\hline WHR & 0.9 & $8.6-1.0$ & 1 & $0.9-1.0$ & 0.9 & $0.9-1.0$ \\
\hline Systolic BP (mmHg) & 127 & $116.5-139.3$ & 131 & $113.5-137.8$ & 126.5 & $119.8-140.5$ \\
\hline Diastolic BP (mmHg) & 86 & $79.8-94.0$ & 86.5 & $80.0-94.3$ & 86 & $79.0-92.0$ \\
\hline PWV (ms) & 9.1 & $8.3-10.2$ & 9.8 & $8.5-10.7$ & $8.7^{*}$ & $8.1-9.7$ \\
\hline Stress & 10 & $4.0-18.0$ & 6 & $6.0-12.0$ & $12.0 * *$ & $8.0-20.0$ \\
\hline Depression & 4 & $4.0-18.0$ & 2 & $0.0-4.0$ & $12.0 * *$ & $4.0-14.0$ \\
\hline Self esteem & 20 & $17.0-24.8$ & 25 & $19.0-27.0$ & $19.0 * *$ & $14.0-20.0$ \\
\hline Emotional eating & 2.9 & $1.8-3.6$ & 2.5 & $1.4-3.2$ & $3.1 * *$ & $2.3-3.8$ \\
\hline External eating & 3.1 & $2.6-3.5$ & 3 & $2.1-3.1$ & $3.2 *$ & $2.9-3.6$ \\
\hline Restrained eating & 2.6 & $2.1-3.2$ & 2.6 & $2.1-3.1$ & 2.7 & $2.1-3.2$ \\
\hline IWQOL & 77.8 & $67.5-87.3$ & 85.9 & $74.4-92.7$ & $74.6 * *$ & $59.1-85.3$ \\
\hline
\end{tabular}

$* P \leq 0.05, * * P \leq 0.01$ (Mann-Whitney $\mathrm{U}$ test).

In conclusion, this study indicates that obese individuals with poor sleep quality are more likely to suffer from depression, stress, low self esteem and are, possibly, more likely to eat in relation to their emotions and external influences. The direction of causality cannot be established from this cross-sectional study; however, the co-existence of these factors reinforces the need for obesity interventions to target and assess all parameters of health.

1. Beccuti G \& Pannain S (2011) Curr Opin Clin Nutr Metab Care 14, 402-412.

2. Knutson K (2010) Best Pract Res Clin Endocrinol Metab 24, 731-741.

3. Slattengren A, Kulie T, Redemer J et al. (2011) J Am Board Fam Med 24, 75-85. 